\title{
Coupled nonlinear oscillations of microbubbles
}

\author{
A. Ooi* $\quad$ R. Manasseh ${ }^{\dagger}$ \\ (Received 30 October 2004, revised 10 February 2005)
}

\begin{abstract}
The coupling effects on the acoustic signature from nonlinear oscillations of a group of microbubbles is investigated. In general, exploring this phenomenon would require solving a set of (linearly) coupled nonlinear ordinary differential equations (ODEs). However, assuming that the initial conditions of all bubbles are identical and that all bubbles are equi-distant from each other simplifies the governing equations to just a single ODE. Numerical data obtained by solving this ODE is used to investigate the effects of bubble population size on the subharmonics and ultraharmonics of the system. As the number of bubbles is increased, the natural frequency and the damping of the system decreases. There is a slight shift in the frequencies at which the maximum bubble oscillation occur. The amplitude of oscillations near the main resonance are significantly reduced as the number of bubble increases.
\end{abstract}

${ }^{*}$ Dept. Mechanical \& Manufacturing Engineering, University of Melbourne, Victoria, Australia. mailto:a.ooi@unimelb.edu.au

${ }^{\dagger}$ CSIRO, Highett, Melbourne, Australia.

See http://anziamj.austms.org.au/V46/CTAC2004/0oi1 for this article, (C) Austral. Mathematical Soc. 2005. Published March 21, 2005. ISSN 1446-8735 


\section{Contents}

1 Introduction

C103

2 Theory

C105

3 Behaviour of unforced system

C107

4 Forced system

C109

5 Conclusions

C114

References

C114

\section{Introduction}

The response of bubbles to an applied external acoustic field and the fields created naturally by bubbles have been known to play an important role in many engineering and medical applications. For example, in microfluidics, the response of microbubbles to an applied sound field has been used to pump or stir liquids $[1,2]$. The acoustic frequency passively emitted by bubbles has been used to measure the size of bubbles inside opaque industrial liquids [3]. In the medical arena, ultrasound contrast agents can be regarded as microbubbles encapsulated with a polymer, protein or lipid shell whose linear and nonlinear acoustic properties make them very useful in medical diagnostic imaging $[4,5]$. There are also exciting prospects for microbubbles to biochemically target certain tissue types. For example, the scattering of ultrasound by targeted microbubbles could be used to diagnose [6, e.g.] and possibly also destroy even tiny metastatic tumors.

For these reasons, many mathematical models have been proposed to study the nonlinear nature of gas-bubble oscillations in a liquid. Most of 
the mathematical models look very similar to the classical Rayleigh-Plesset equation

$$
\begin{aligned}
\rho\left(R \ddot{R}+\frac{3}{2} \dot{R}^{2}\right)= & \left(\frac{2 \sigma}{R_{0}}+P_{0}-P_{v}\right)\left(\frac{R_{0}}{R}\right)^{3 \kappa} \\
& +P_{v}-P_{0}-\frac{2 \sigma}{R}-4 \mu \frac{\dot{R}}{R}-P_{\text {ext }}(t),
\end{aligned}
$$

where $R(t)$ is the instantaneous bubble radius, $R_{0}$ is the bubble radius at equilibrium, $\mu$ is the viscosity of the liquid, $\rho$ is the density of the liquid, $\sigma$ is the surface tension of the liquid and $\kappa$ is the polytropic exponent of the gas inside the bubble. Only air bubbles in water will be considered here so all simulations are carried out with $\rho=1000 \mathrm{~kg} / \mathrm{m}^{3}, \mu=0.001 \mathrm{~kg} / \mathrm{m} / \mathrm{s}$, $\kappa=1.33, P_{v}=2330 \mathrm{~Pa}, P_{0}=100000 \mathrm{~Pa} . P_{\text {ext }}(t)$ is the imposed external pressure field and it is typically a known function of time. Owing to the nonlinear nature of the governing equations, it is difficult to obtain analytical solutions. Numerical methodology must be employed [7] to provide insights into the physics of the problem.

In most of the studies on nonlinear bubble oscillations carried out thus far, the bubbles are assumed to be sufficiently far apart and the effects due to bubble interactions have been neglected. The integrated properties (for example, radiated pressure) of a cluster of bubbles are then calculated by simply summing up the contributions of individual bubbles [8, e.g.]. However, recently Manasseh [9] has shown that if the bubbles are close to one another, coupling effects become significant and cannot be neglected.

Here the coupling effects on the acoustic signature from nonlinear oscillations of a group of microbubbles are investigated. This involves solving a set of (linearly) coupled nonlinear ordinary differential equations. Data obtained from the numerical computations will be used to investigate the frequency response of equally sized microbubbles as the number of bubbles in the population is increased. Analyses on the effects of population size on the subharmonics and ultraharmonics are discussed. 


\section{Theory}

Despite several limitations, the classical Rayleigh-Plesset equation (1) is one of the most widely used models in the simulation of radial bubble oscillations. The properties of this model has been extensively investigated [7]. One major drawback of this model is that it assumes the speed of sound is infinite in the liquid, which could be an issue if the group of bubbles spans a significant fraction of the wavelength of sound. This mathematical model also neglects effects of heat and mass transfer and gas dissociation that could be significant at large oscillation amplitudes. More complex models have been proposed and Vokurka [10] has performed a detailed analysis on the various advantages and disadvantages of the many different models. Here we address cases where the bubbles are close, so the liquid sound speed are neglected, and oscillation amplitudes are not extreme; thus Eq. (1) is a reasonable starting point.

To account for the effects of other nearby bubbles, a coupled-oscillator approximation is used, similar to that of previous analyses of linearly coupled pairs of linearly-oscillating bubbles [11, 12, 13, 14, e.g.] but for an arbitrary number $N_{\text {bub }}\left[9\right.$, e.g.]. Effectively, the $P_{\text {ext }}$ term in Eq. (1) is modified by the pressure scattered by the other bubbles [13, e.g.] as,

$$
P_{s i}=\sum_{j \neq i}^{N_{\mathrm{bub}}} \frac{\rho}{s_{i j}} \frac{d}{d t}\left(R_{j}^{2} \dot{R}_{j}\right),
$$

where $s_{i j}=s_{j i}$ is the distance of bubble $i$ from bubble $j$. The total external pressure on bubble $i$ is then

$$
P_{\text {ext }, i}=P_{s i}+P_{A, i}(t) .
$$

where $P_{A, i}(t)$ is the applied pressure of any external field on bubble $i$. Combining Eqs. (1) and (3) gives the following coupled governing equation for 
coupled bubble oscillations,

$$
\begin{aligned}
\rho R_{i} \ddot{R}_{i}+\rho \frac{3}{2} \dot{R}_{i}^{2}= & \left(\frac{2 \sigma}{R_{0}}+P_{0}-P_{v}\right)\left(\frac{R_{0}}{R_{i}}\right)^{3 \kappa}+P_{v}-P_{0}-\frac{2 \sigma}{R_{i}}-4 \mu \frac{\dot{R}_{i}}{R_{i}} \\
& -\sum_{j \neq i}^{N_{\text {bub }}} \frac{\rho}{s_{i j}} \frac{d}{d t}\left(R_{j}^{2} \dot{R}_{j}\right)-P_{A, i}(t) .
\end{aligned}
$$

We assume that $s_{i j}=D=$ constant. Thus the distance of any bubble to any other bubble in the bubble population is constant. For $N_{\text {bub }}=3$ this situation corresponds to a group of bubbles arranged at the vertices of an equilateral triangle. For $N_{\text {bub }}=4$, the bubbles are placed at the vertices of an equilateral tetrahedron. Note that it is not physically possible to have a situation where $N_{\text {bub }}>4$ for equi-spaced bubbles.

We further assume that the same external driving pressure field acts on all the bubbles, that is, $P_{A, 1}(t)=P_{A, 2}(t)=P_{A, 3}(t)=P_{A, 4}(t)=P_{A}(t)$, then $R_{i}(t)=R_{j}(t)=R(t)$. Substituting into Eq. (4) with simple algebraic manipulation gives

$$
\begin{aligned}
& \rho R \ddot{R}+\left(N_{\mathrm{bub}}-1\right) \frac{\rho}{D}\left(R^{2} \ddot{R}\right) \\
= & -\rho \frac{3}{2} \dot{R}^{2}+\left(\frac{2 \sigma}{R_{0}}+P_{0}-P_{v}\right)\left(\frac{R_{0}}{R}\right)^{3 \kappa}+P_{v}-P_{0} \\
& -\frac{2 \sigma}{R}-4 \mu \frac{\dot{R}}{R}-\left(N_{\mathrm{bub}}-1\right) \frac{2 \rho}{D}\left(R \dot{R}^{2}\right)-P_{A}(t) .
\end{aligned}
$$

Equation (5) represents the idealized case where all the bubbles are equally spaced, have the same initial conditions and are excited by the same external pressure field. Hence, all bubbles have the same radius and the results presented here would be directly comparable only with experiments in a very specifically designed microfluidic system, rather than experimental data from a group of contrast-agent microbubbles in vivo. However, the results may be used as both an ideal reference for a microtechnological application, and to 
show the possible trends of a contrast-agent system as the number of bubbles is increased from one towards a number that is more typical in medical applications.

Equation 5 is linearised by setting $R(t)=R_{0}+\epsilon(t)$ and ignoring higher order terms in $\epsilon(t)$, giving,

$$
\begin{aligned}
& \left(1+\left(N_{\mathrm{bub}}-1\right) \frac{R_{0}}{D}\right) \ddot{\epsilon}+\left(\frac{4 \mu}{\rho R_{0}^{2}}\right) \dot{\epsilon} \\
+ & \left(\frac{3 \kappa}{\rho R_{0}^{2}}\left(P_{0}+P_{v}\right)+(3 \kappa-1) \frac{2 \sigma}{\rho R_{0}^{3}}\right) \epsilon=0 .
\end{aligned}
$$

Thus, in the absence of damping, the linear resonance frequency for this bubble system is

$$
f_{0}=\frac{1}{2 \pi} \sqrt{\frac{\frac{3 \kappa}{\rho R_{0}^{2}}\left(P_{0}+P_{v}\right)+(3 \kappa-1) \frac{2 \sigma}{\rho R_{0}^{3}}}{1+\left(N_{\text {bub }}-1\right) \frac{R_{0}}{D}}} .
$$

\section{Behaviour of unforced system}

This initial investigation analyses the behaviour of Eq. (5) for cases where $P_{A}(t)=0$. Eq. (5) is a nonlinear second order ordinary differential equation with one stationary point occurring at $\dot{R}=0$ and $R=R_{0}$. Following [15], the qualitative behaviour of such a system is studied by plotting the trajectories in the phase plane (with coordinates $R(t)$ and $\dot{R}$ ). The phase portrait for the system for $R_{0}=10 \mu \mathrm{m}$ is shown in Figure 1. The trajectories represent a stable clockwise spiral illustrating that in the absence of any external forcing, the radius of the bubble will eventually tend to rest $(\dot{R}=0)$ at $R=R_{0}$. The qualitative behaviour of the system does not appear to be dependent upon $N_{\text {bub }}$. Eq. (6) indicates that the amount of damping decreases with increasing $N_{\text {bub }}$. This is illustrated in Figure 2 which shows the temporal 


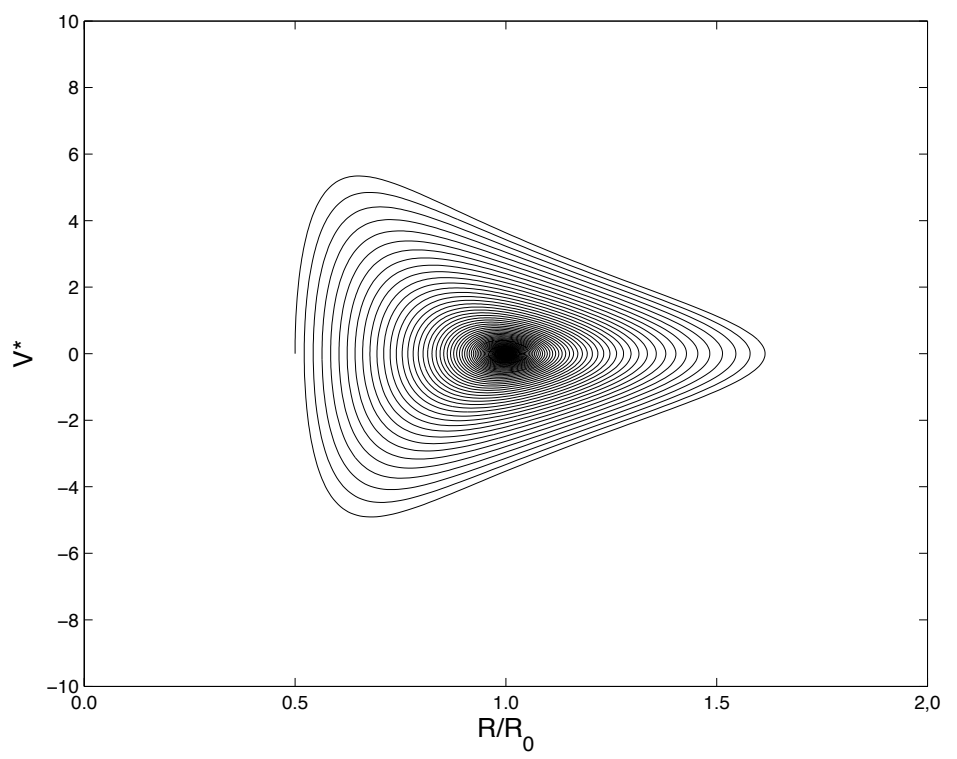

FIgURE 1: Phase portrait of the system defined by Eq. (5). $V^{*}=$ $\dot{R} /\left(R_{0} / T\right)$.
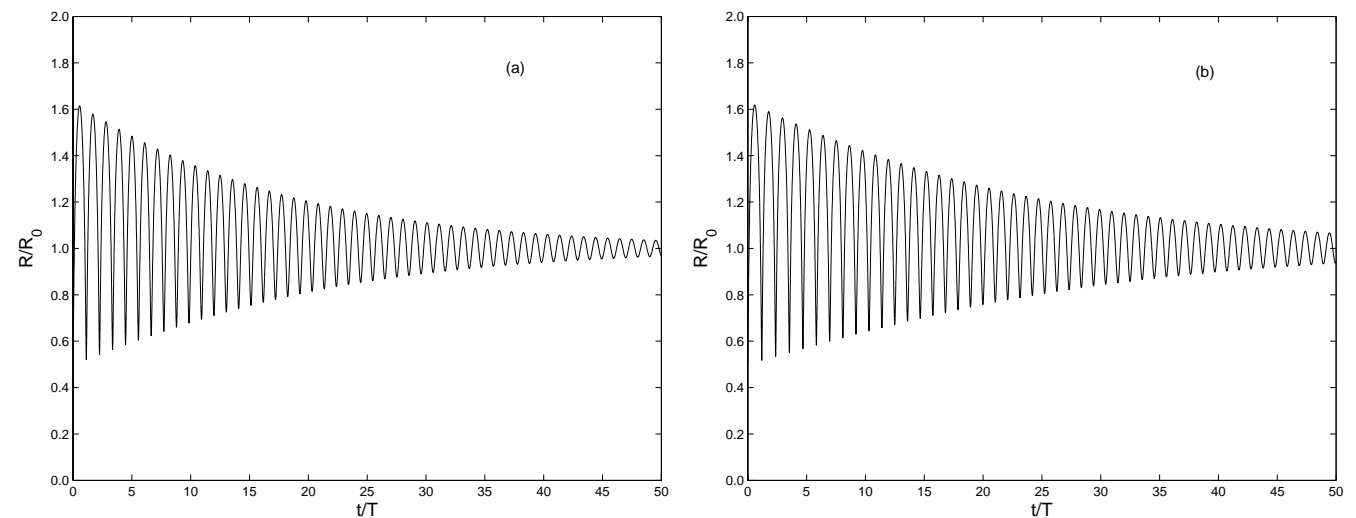

Figure 2: The evolution of $R(t)$ for a freely oscillating bubble with $R_{0}=$ $10 \mu \mathrm{m}$. (a) $N_{\text {bub }}=1$ and (b) $N_{\text {bub }}=4$ 

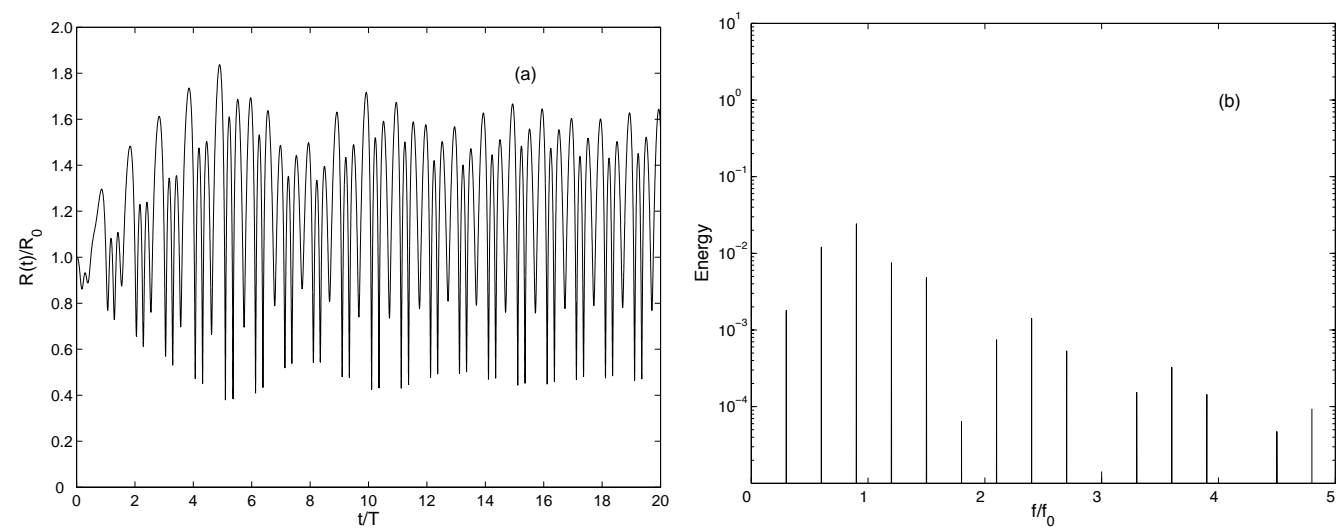

Figure 3: Single bubble response. (a) $R(t) / R_{0}$ for $R_{0}=10 \mu \mathrm{m}$. The pressure amplitude, $\alpha=0.6$ bar and frequency, $f_{\text {ext }} / f_{0}=0.3$. (b) Corresponding Fourier spectra of the steady-state oscillation.

evolution of $R(t)$ with $N_{\text {bub }}=1$ and 4 . The initial conditions for the simulation were $R(t=0)=0.5 R_{0}$ and $\dot{R}(t=0)=0$. Visual comparison of the figures clearly shows that there is a higher damping for the system with $N_{\text {bub }}=1$.

\section{Forced system}

In the simulations described below, the bubble system will be forced by the oscillating pressure field $P_{A}=\alpha \sin \left(2 \pi f_{\text {ext }} t\right)$, where $\alpha$ and $f_{\text {ext }}$ are the amplitude and frequency of the external pressure field respectively. In all simulations discussed here, the system is initialized with $R(t=0)=R_{0}$ and $\dot{R}(t=0)=0$. There are two different timescales for the simulations described here. One is the period of the linearized equations $\left(T_{0}=1 / f_{0}\right)$, and the other one is the period of the forced oscillations $\left(T_{\text {ext }}=1 / f_{\text {ext }}\right)$. We limit our scope of study to cases where $\alpha=0.6$ bar and $N_{\text {bub }}=1,2,3$ 
and 4 . Figure 3 shows a typical time series for one of our simulations with $N_{\text {bub }}=1$. The radius $R(t)$ goes through an initial transient phase before settling down to a steady-state oscillation. This behaviour is typical for all the simulations discussed here and is in agreement with the single-bubble calculation of Lauterborn [7] for the some parameters. If the applied pressure amplitude $\alpha$ is too large, then $R(t)$ undergoes wild fluctuations and does not settle down to a periodic behaviour. It is well documented in the extensive literature on cavitation and sonoluminescence [16, for references therein, e.g.] that such behaviour represents extremes for which the simple RayleighPlesset equation (1) requires modification. The data in Figure 3(a) shows that periodic steady state oscillations occur at approximately $t / T>15$, where

$$
T=\max \left(T_{0}, T_{\text {ext }}\right) .
$$

In many of our simulations, the initial transients take a much longer time to die out. Sometimes, steady-state periodic oscillations only occur when $t / T>150$. The energy spectra for the steady-state signal is shown in Figure 3(b). Most of the energy occurs in discrete frequencies. This behaviour is typical in all the data reported in this paper. The frequency response curves, that is, the plot of $\left(R_{\max }-R_{0}\right) / R_{0}$ versus $f_{\text {ext }} / f_{0}$ is shown in Figure 4, where $R_{\max }$ is the maximum radius of the bubble during its steadystate oscillation. Data samples for the calculation of $R_{\max }$ were only taken from $200<(t / T)<250$ to ensure that any effects from the initial transients can be ignored. The frequency response curves for a bubble system driven with a pressure amplitude $\alpha=0.6$ bar and with $R_{0}=10 \mu \mathrm{m}$ and $D=50 \mu \mathrm{m}$ for $N_{\text {bub }}=1,2,3$ and 4 is shown in Figure 4 . Following the terminology of Lauterborn [7], the peaks labelled 'A' represent the main harmonics, the subharmonic is labelled ' $C$ ' and the ultraharmonics are labelled 'B'. Similarly to the finding of Lauterborn [7] for a single bubble, the main resonance for our multi-bubble case is in the region of $f_{\text {ext }} / f_{0}=1$. The main resonance peak is asymmetric: it 'leans' towards lower frequencies and there is a sudden discontinuity in the plot as $f_{\text {ext }}$ is lowered. Figure 5 shows the close-ups of two regions of Figure 4, that is, in the vicinity where $f_{\text {ext }} / f_{0}=10^{-0.1} \approx 0.7943$ 


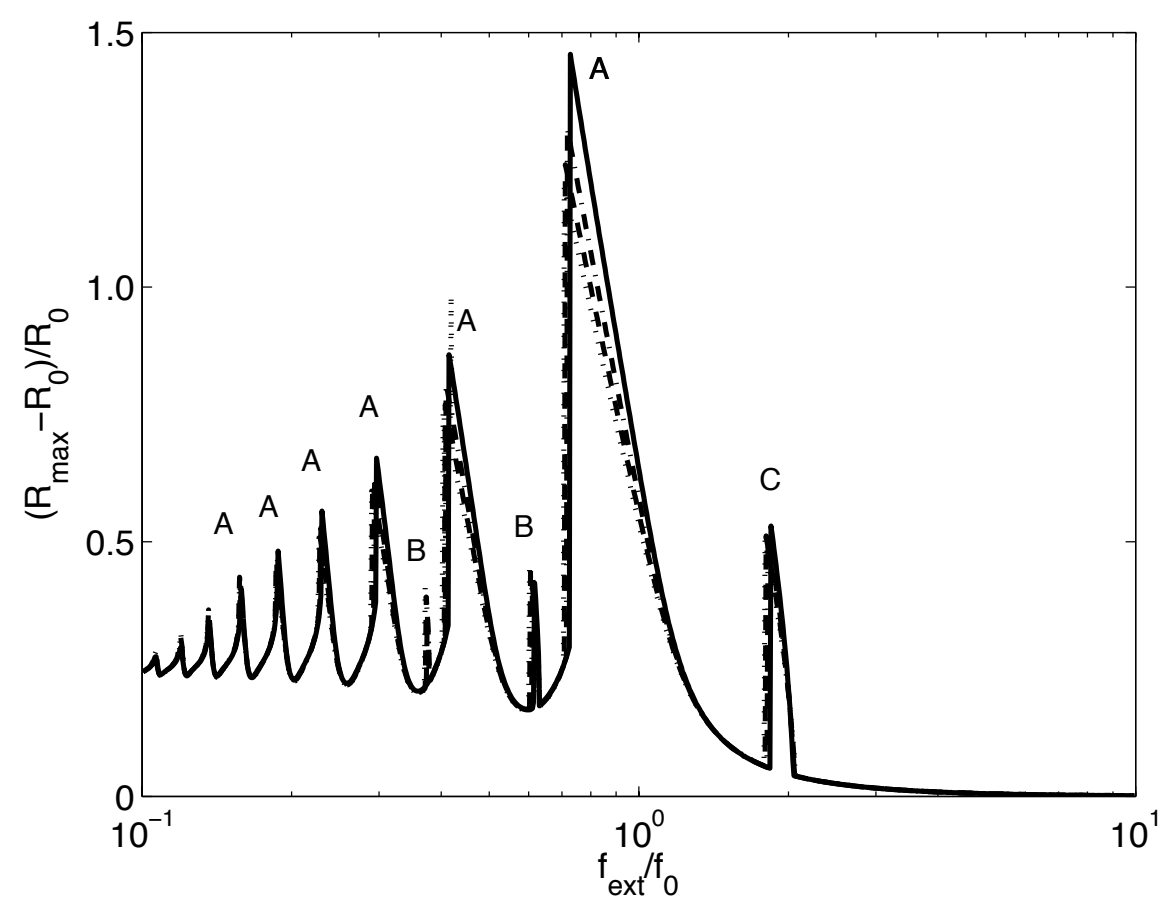

FiguRE 4: Frequency response curves for $R_{0}=10 \mu \mathrm{m}$ and the separation between bubbles, $D=50 \mu \mathrm{m}$. $-N_{\text {bub }}=1,--N_{\text {bub }}=2,-\cdots N_{\text {bub }}=$ $3, \cdots N_{\text {bub }}=4$. 

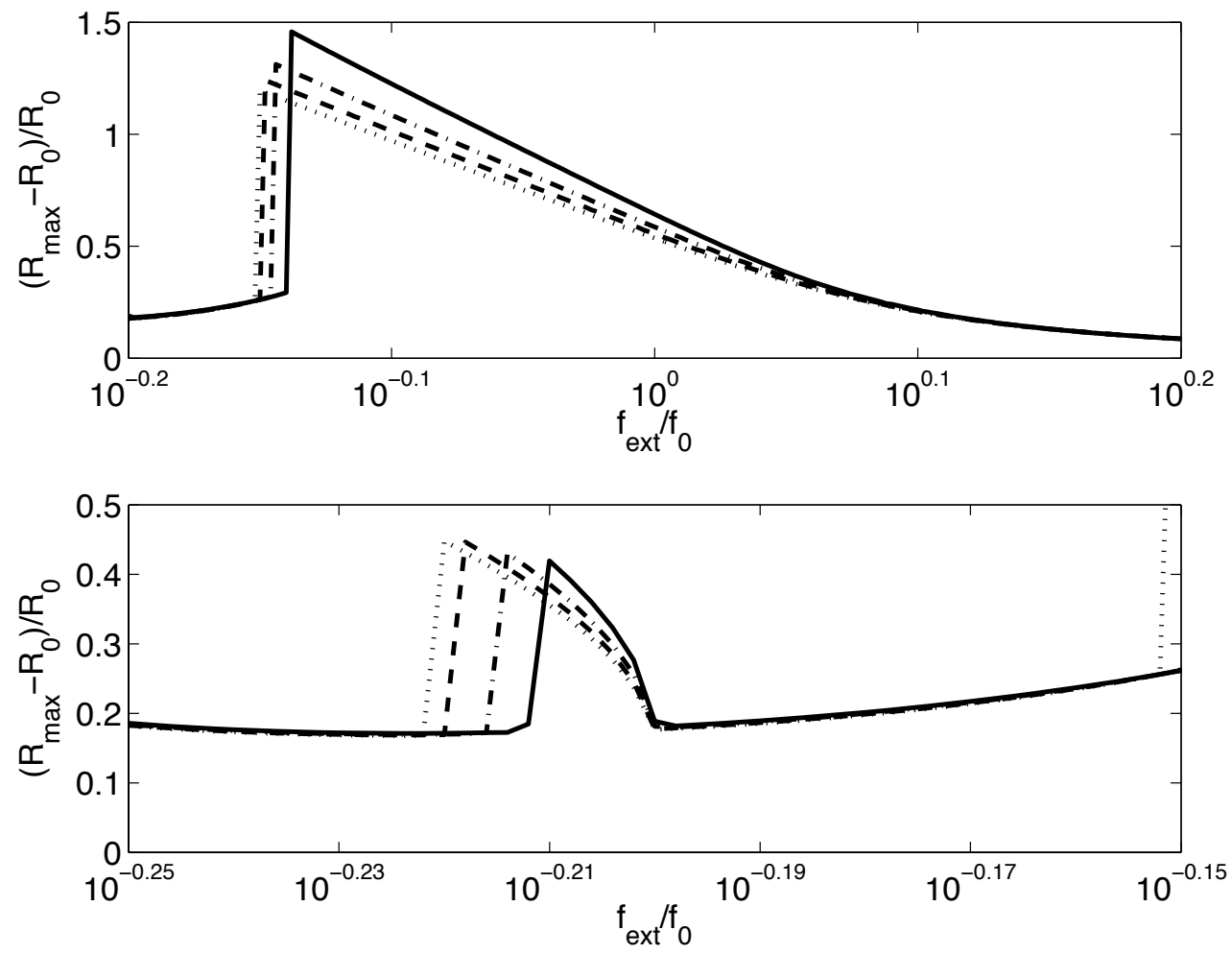

Figure 5: Close-ups of frequency response curves of two regions of Figure 4. Top panel: $f_{\text {ext }} / f_{0}=10^{-0.1} \approx 0.7943$; Bottom panel: $f_{\text {ext }} / f_{0}=10^{-0.21} \approx$ 0.6166. All parameters as for Figure 4. Note different vertical scales in top and bottom panels. 

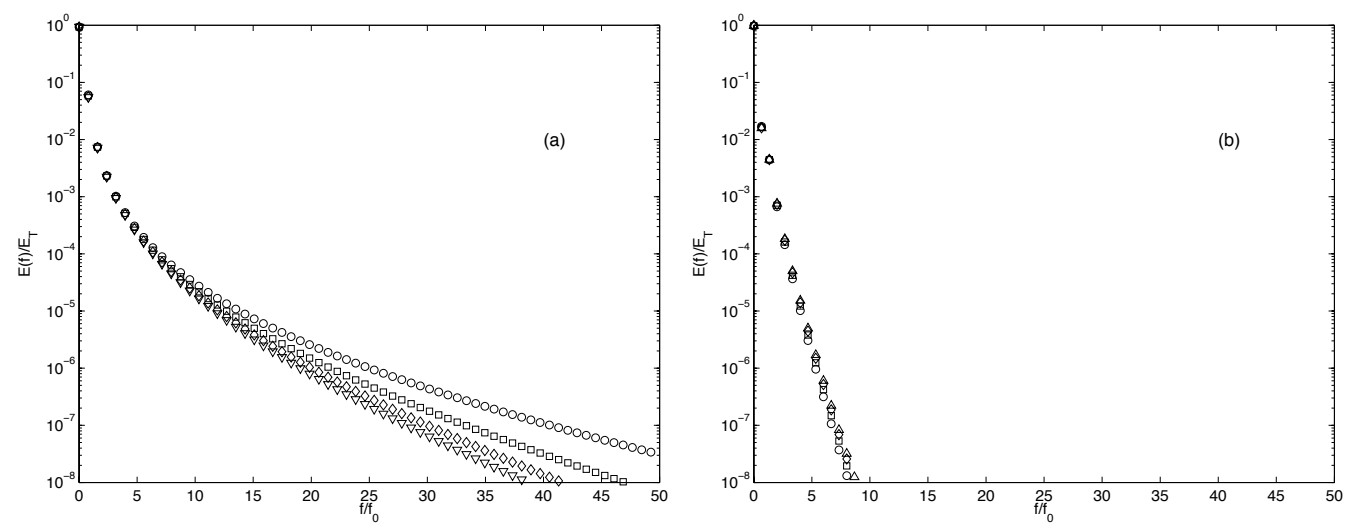

FiguRE 6: Energy spectra of signal: (a) $f_{\text {ext }} / f_{0}=10^{-0.1}$; (b) $f_{\text {ext }} / f_{0}=$ $10^{-0.21} . \alpha=0.6$ bar and $R_{0}=10 \mu \mathrm{m}$ and $D=50 \mu \mathrm{m} . \quad \circ N_{\text {bub }}=1$, $\square N_{\text {bub }}=2, \diamond N_{\text {bub }}=3, \nabla N_{\text {bub }}=4$.

(in the vicinity of a harmonic, the main resonance) and $f_{\text {ext }} / f_{0}=10^{-0.21} \approx$ 0.6166 (in the vicinity of an ultraharmonic).

Now the effect of the presence of more than one bubble is clear. The frequency response curves in Figure 5 show different behaviours as $N_{\text {bub }}$ is varied. In the vicinity of the main harmonics, (that is, the peaks labelled ' $\mathrm{A}$ '), as we lower the value of $f_{\text {ext }}$, the value of $R_{\max }$ gets smaller. However, in the vicinity of the ultraharmonics, (that is, the peaks labelled 'B'), as we lower the value of $f_{\text {ext }}$, the opposite trend is observed.

The energy spectra for the simulation computed with $f_{\text {ext }} / f_{0}=10^{-0.1}$ and $f_{\text {ext }} / f_{0}=10^{-0.21}$ is shown in Figures 6 . In the region of the main harmonics, Figure 6(a) shows a definite trend that as we increase $N_{\text {bub }}$, the energy at higher frequencies decreases. However, close to the ultraharmonics, Figure 6(b) shows that the energy spectra are not so sensitive to the number of bubbles in the system. 


\section{Conclusions}

The governing equation for a system of equally sized bubbles at the same distance from each other was derived; it is valid for a system with four or less bubbles. Analyses found that the natural frequency and the damping of the system decreases as the number of bubbles increases. Numerical simulations close to the main harmonic of the system show that there is a strong effect on the high frequency components of the signal. However, close to the ultraharmonics the energy spectra are relatively insensitive to the number of bubbles in the system. As the number of bubbles is increased, the response curves at the main resonance and the ultraharmonic shift, decreasing the main resonance but slightly increasing its ultraharmonic.

\section{References}

[1] Yuan, H., Prosperetti, A., Popinet, S., Zaleski, S. Growth and collapse of a vapor bubble in a narrow tube J. Acoust. Soc. Am., 106 (2): 674-681, 2000. C103

[2] Liu, R., Yang, J., Leningk, R., Bonanno, J., Grodzinski, P. Self-contained, fully integrated biochip for sample preparation, polymerase chain reaction amplification, and DNA microarray detection Anal. Chem., 76: 1824-1831, 2004. C103

[3] Manasseh, R., LaFontaine, R. F., Davy, J., Shepherd, I. C., and Zhu, Y. Passive acoustic bubble sizing in sparged systems. Exp. Fluids, 30(6):672-682, 2001. C103

[4] Hilgenfeldt, S., Lohse, D., Zomack, M. Response of bubble to diagnostic ultrasound: a unifying theoretical approach. Euro. Physical J. B, 4: 247-255, 1998. C103 
[5] J. Allen, D. Kruse, P. Dayton, and K. Ferrara. Effect of coupled oscillations on microbubble behaviour. J. Acoust. Soc. Am., 114(3):1678-1690, 2003. C103

[6] Frauscher, F., Klauser, A., Halpern, E., Detection of prostate cancer with a microbubble ultrasound contrast agent. Lancet, 357: 1849-1850, 2001. C103

[7] W. Lauterborn. Numerical investigation of nonlinear oscillations of gas bubbles in liquids. J. Acoust. Soc. Am., 59(2): 283-293, 1976. C104, C105, C110

[8] N. de Jong, R. Cornet and C. Lancee. Higher harmonics of vibrating gas-filled microspheres. Part one: simulations. Ultrasonics., 32(6):447-453, 1994. C104

[9] R. Manasseh, A. Nikolovska, A. Ooi, and S. Yoshida. Anisotropy in the sound field generated by a bubble chain. To appear in J. Sound and Vibration, 2004. C104, C105

[10] K. Vokurka Comparison of Rayleigh's, Herring's and Gilmore's Models of Gas Bubbles. Acustica, 59:214-219, 1986. C105

[11] Tolstoy, I. Superresonant systems of scatterers. i. J. Acoust. Soc. Am., 80(1):282-294, 1986. C105

[12] Doinikov, A. A. and Zavtrak, S. T. On the mutual interaction of two gas bubbles in a sound field. Phys. Fluids, 7(8): 1923-1930, 1995. C105

[13] R. Mettin, I. Akhatov, U. Parlitz and C. D. Ohl and W. Lauterborn. Bjeknes forces between small cavitation bubbles in a strong acoustic field. Physical Review E, 56(3):2924-2931, 1997. C105

[14] Ida, M. A characteristic frequency of two mutually interacting gas bubbles in an acoustic field. Physics Letters A, 297(3-4): 210-217, 2002. C105 
[15] J. Keller and M. Miksis. Bubble oscillations of large amplitude. J. Acoust. Soc. Am., 68(2):628-633, 1980. C107

[16] Hilgenfeldt, S., Siegfried, G., Lohse, D. A simple explanation of light emission in sonoluminescence. Nature, 398: 402-405, 1999. C110 